\title{
Device for the Control of Oxygen Concentration in Multiwell Cell Culture Plates
}

\author{
Shawn C. Oppegard and David T. Eddington
}

\begin{abstract}
Oxygen is a key modulator of many cellular pathways but current devices permitting in vitro oxygen modulation fail to meet the needs of many researchers. In this study, a microfabricated insert for multiwell formats has been developed to control the gas concentration of each well independent of the global incubator's condition. The platform consists of a polydimethylsiloxane (PDMS) insert that nests into a standard multiwell plate and serves as a passive network with a gas permeable membrane aimed to deliver gas to adherent cell cultures. Preliminary data demonstrate that the insert is effective in controlling the oxygen concentration at the cell surface inside a well with equilibration times in minutes rather than hours for conventional technologies. A wide variety of oxygen profiles can be attained based on the device design, such as the cyclic profile achieved in this study, and even gradients in local oxygen concentration to mimic those found in vivo for more biomimetic cellular models.
\end{abstract}

\section{INTRODUCTION}

Oxygen is a key metabolic variable that influences many different biological phenomena, however current tools to probe this variable are crude and inefficient and have not changed since the dawn of cell culture techniques. The hypoxic chamber remains as the tool of choice and has seen a great deal of use in applications requiring control over the oxygen tension exposed to cells[1-3]. However, the device fails to satisfy the needs of biomedical researchers to efficiently modulate oxygen tensions over multiple conditions as a separate chamber is required per condition. Oxygen is increasingly implicated in many signaling pathways and one route of modulation is through the hypoxia-inducible factor (HIF) family of heterodimeric transcription factors which regulate the cellular response to oxygen tension [4]. HIF-1alpha is a ubiquitous transcription factor that has been found to influence development [4], solid tumor growth [4], and angiogenesis [5]. Enabling a simple tool to explore this would greatly facilitate these investigations and accelerate scientific discovery. In addition to increasing the throughput of hypoxic experimentation, the platform has potential to facilitate new and exciting experiments not possible with current techniques. Gradients of oxygen are standard byproducts of

Manuscript received April 5, 2009. This work was supported in part by the U.S. Department of Public Health.

S. C. Oppegard is a graduate student at the University of Illinois at Chicago, Chicago, IL 60607 USA (e-mail: soppeg2@uic.edu)

David T. Eddington is an assistant professor in the Bioengineering Department, University of Illinois at Chicago, Chicago, IL 60607 USA cellular metabolism found throughout every tissue and across all organisms. These gradients of oxygen are increasingly highlighted as crucial metabolic regulators when studying drug toxicity [6], the hematopoietic stem cell niche [7], plant biology [8], liver zonation [9, 10], and throughout developmental biology $[11,12]$. Thus replicating these in vivo gradients in an in vitro model accessible to any standard cell biology lab would have a huge impact across many fields. Several groups have developed devices for controlling the oxygen tension experienced by cell cultures that are aimed to improve upon the hypoxic chamber $[6,13$ 16]. Unfortunately, these devices require very specific parameters for operation, including the need for complex fluid handling, and even an electric current to introduce oxygen via electrolysis [16]

A new device with more precise temporal and spatial control over gas concentrations experienced by the cell, while possessing a smaller lab footprint than other current cell oxygenation devices, could be very beneficial to the scientific community. To meet this demand a new device has been fabricated consisting of a PDMS insert that nests into a standard multiwell plate as shown in Figure 1a. The insert contains a series of pillars matching the number and spacing of wells of the plate it is designed to nest into. The work described in this manuscript focuses on a 6-well version, however the concept can be expanded to higher density multiwell plates (e.g. 12, 24, 48 or 96-wells). Oxygen is injected through microchannels embedded at the base of each pillar and is separated from the fluidic contents of the culture well by a $0.1 \mathrm{~mm}$ gas permeable PDMS membrane. The bottom of the membrane can be fixed at various distances from the substrate of the well through employing spacers or by altering the length of the pillar. The oxygen microchannels connect to gas cylinders which provide the pressure to deliver the gas throughout the insert. The insert is a passive network with a gas permeable membrane aimed to deliver gas to adherent cell cultures in multiwell plates. The insert acts as a sink or source of oxygen depending on the concentration of the oxygen in the microchannels and is immersed in the cell media of the well. The gas is delivered to the cell monolayer through simple diffusion of oxygen across the PDMS membrane and dissolves into the culture media of the multiwell plate. The oxygenation routing and pillar microchannels is fabricated

(corresponding author to provide phone: 312-355-3278; fax: 312-996-5921; e-mail: dte@uic.edu). 
through standard soft lithographic techniques [17] and the pillar array is fabricated through casting PDMS into a machined Delran mold. A schematic cross section of a representative pillar in a well is shown in Figure $1 \mathrm{~b}$ and depicts the cell monolayer at the bottom of the well positioned approximately $200 \mu \mathrm{m}$ from the bottom of a pillar. This close spacing between the PDMS membrane and cell monolayer allows for rapid diffusion to impose steady state gradients of oxygen across the well within minutes and is not altered by the slight deflection of the elastomeric flexible membrane. Many oxygenation profiles can be generated depending on the layout of the microfluidic oxygenation channels in the base of the pillar.
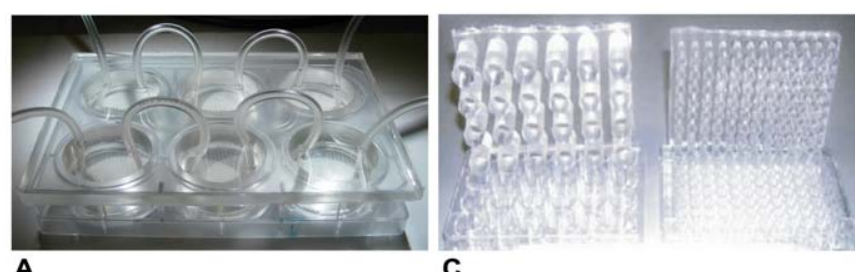

A
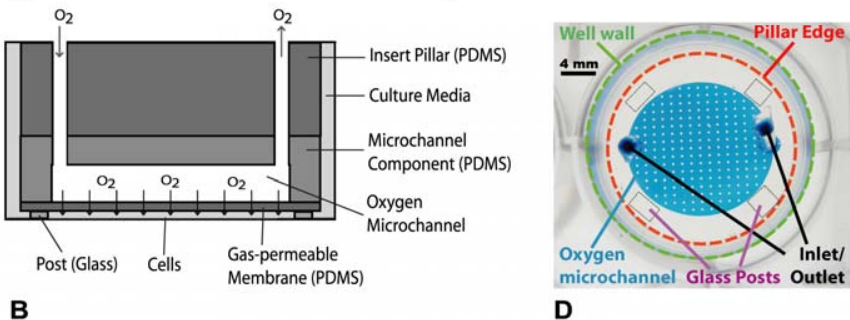

Figure 1. Oxygen insert device. A) Oxygen device nested into a 6-well plate. B) Cross-sectional schematic of pillar. Oxygen flows into the device through the inlet and travels across a microfluidic network at the bottom of the pillar. Oxygen can freely diffuse across the gas-permeable PDMS membrane and dissolve into the culture media. C) Examples of 24 and 96well pillar arrays. D) Macroscope image showing the various features of a single-channel pillar from above.

PDMS is used as the material of the insert due to its optical clarity, gas permeability $\left(\mathrm{D}_{\text {oxygen in }} \mathrm{B}_{\mathrm{PDMS}}=3 \times 10^{-5}\right.$ $\mathrm{cm}^{2} / \mathrm{s}$ ), and ease of rapid prototyping. However, several recent studies have highlighted the ability of PDMS to absorb water and hydrophobic soluble factors [18, 19]. Only the membrane component of our platform needs to be made with PDMS due to its excellent oxygen permeability and the bulk of our platform (including the gas microchannels and pillar array) can be fabricated with alternative polymers more resistant to hydration and absorption such as polymethylmethacrylate or polycarbonate. Our initial studies rely on PDMS due to the ease of rapid prototyping and we have taken measures to avoid hydration of the manifold from altering the experimental conditions. Water loss through absorption into PDMS would be detrimental in cell culture that relies on specific concentrations of solutes to maintain $\mathrm{pH}$ and nutrient levels. Specifically the device is pre-hydrated through immersion in cell media or water prior to experimentation. While PDMS is not an ideal material for cellular studies, measures can be taken to minimize the effects such as pre-hydration of the device or extraction of unpolymerized polymer.

\section{MATERIALS AND METHODS}

\section{A. Device Fabrication}

The hypoxia device presented here consists of three main components: 1) the gas permeable membrane, 2) the microfluidic network component, and 3) the insert scaffold. The gas permeable membrane is fabricated by defined spinning of a 10:1 ratio of PDMS prepolymer and curing agent (Sylgard 184 kit, Dow Corning) on a silicon wafer using a Laurel spinner to yield $100 \mu \mathrm{m}$ thickness. The microfluidic network was made using standard SU-8 photolithography and PDMS replica molding. The insert scaffold was fabricated by molding PDMS in a machine polycarbonate scaffold. Components were bonded using oxygen plasma treatment. For some of the oxygen validation studies, cut glass posts were bonded to the membrane to alter the spacing between the membrane and the well bottom.

\section{B. Oxygen concentration validation with the device}

Oxygen tension within each well was characterized using a planar oxygen sensor (FOXY-SGS, Ocean Optics) which contains a ruthenium complex whose fluorescence is quenched in the presence of oxygen. Briefly, the slide was placed in the wells of a 6-well plate, simulating where the cells would be present. Oxygen concentrations of $0 \%, 10 \%$, and $21 \%$ (all $5 \% \mathrm{CO}_{2}$, balanced nitrogen tanks) were introduced into the channels of the device and oxygen concentration was measured via fluorescence. Calibration curves at each slide position were first formulated using a custom device that exposed the FOXY slide directly to the introduced gases. Two linear curve equations were fitted to the three data points; one from $0 \%$ to $10 \%$ percent oxygen, and the other from $10 \%$ to $21 \%$ oxygen. For the $0 \%$ oxygen equilibration studies, cut-glass posts were used to establish slide-membrane separations of $0.17 \mathrm{~mm}, 0.47 \mathrm{~mm}$, and 1 $\mathrm{mm}$ to assess the effect of oxygen diffusion distance on measured oxygen concentration at the well surface. $0 \%$ oxygen was introduced into the channel and fluorescence intensity measurements were taken every 10 seconds for 30 min. A hypoxic chamber was also used as a comparison to demonstrate the effectiveness of the device. Briefly, the hypoxic chamber was rapidly flushed with $0 \%$ oxygen and then a steady influx of $0 \%$ oxygen was maintained for $3 \mathrm{~h}$. Additionally, a cyclic profile device was fabricated with two interdigitated and winding microchannels of $0.5 \mathrm{~mm}$ width extending across the pillar to generate a cyclic oxygen profile. Four images were taken at low magnification spanning the entire channel width, intensity linescans were taken across each image, and the data was stitched together. 


\section{Western analysis of HIF-1alpha}

U2OS cells (a human osteosarcoma cell line) were plated at $80-90 \%$ confluency in a 6-well plate and exposed to hypoxia and normoxia using the hypoxic insert device. The cell-seeded plate was placed in the incubator and the device was nested and exposed to either $0.5 \%$ oxygen (hypoxia), $21 \%$ oxygen, or $10 \mu \mathrm{M} \mathrm{CoCl}_{2}$ as a control. $\mathrm{CoCl}_{2}$ has been previously shown to mimic the hypoxic state and as a result cause induction of HIF-1 $\mu$ [20]. After 24 hours, cell lysates were immediately harvested using lysis buffer. Lysate protein was separated on an SDS-Page gel and HIF-1 $\alpha$ was detected using the mouse monoclonal H1alpha67 antibody.

\section{RESULTS}

\section{A. Oxygen concentration validation}

The equilibration experiments demonstrated that the device can rapidly change the oxygen concentration at the well surface (figure 2a). For the $0.17 \mathrm{~mm}$ media distance, the device equilibrated to less than $0.5 \%$ oxygen in about 1.5 min. Equilibration took slightly longer and was less complete for the 0.47 gap distance, taking about $5 \mathrm{~min}$ to achieve roughly $1 \%$ oxygen. For the $1 \mathrm{~mm}$ media distance, the device equilibrated to about $2 \%$ oxygen in about $15 \mathrm{~min}$. Equilibration for the hypoxic chamber was orders of magnitude slower, taking about $3 \mathrm{~h}$ to reach approximately $2.5 \%$ oxygen [21]. Thus, the device is not only significantly faster at equilibrating but also generates oxygen concentrations much lower than conventional methods.

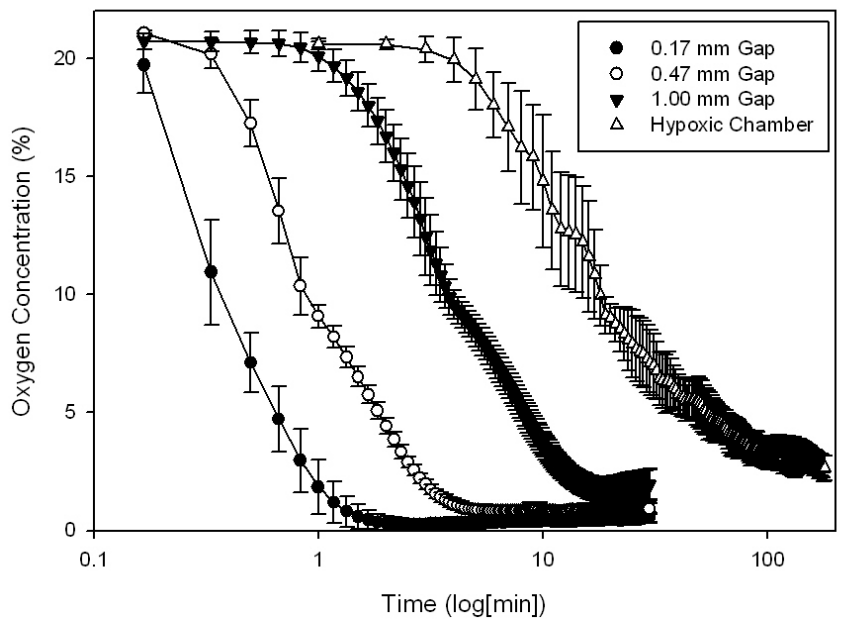

Figure 2. Plot illustrating the effect of post height, and thus oxygen diffusion distance between the membrane and cells, on the equilibration time and effectiveness. Note that the time axis is on a log scale. All three post sizes yield equilibration times much improved over the hypoxic chamber.

The cyclic profile device did indeed generate a cyclic profile as shown in figure 3 . Oxygen can diffuse across the walls separating the winding microchannels and thus limits the extent of hypoxia and hyperoxia.

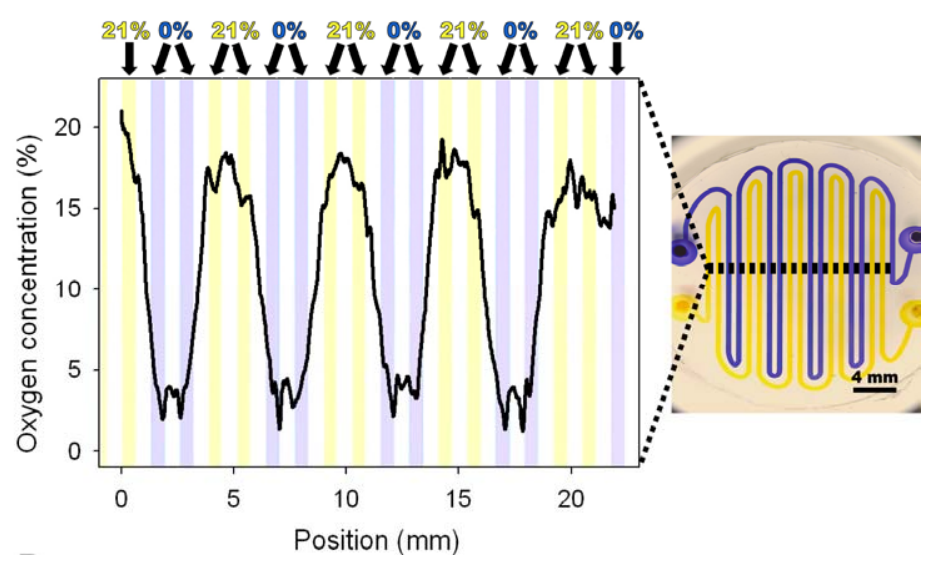

Figure 3. An interdigitated and winding pattern of $500 \mu \mathrm{m}$ width microchannels extending across the pillar results in a cyclic oxygen profile. Dye is used to simulate the two different gas concentrations ( $0 \%$ and $21 \%)$ flowing through the channels.

\section{B. Modulation of transcription factor expression}

Western analysis demonstrated that HIF-1 $\alpha$ was upregulated in U2OS cells exposed to hypoxia as compared to the control cells as shown in figure 4.

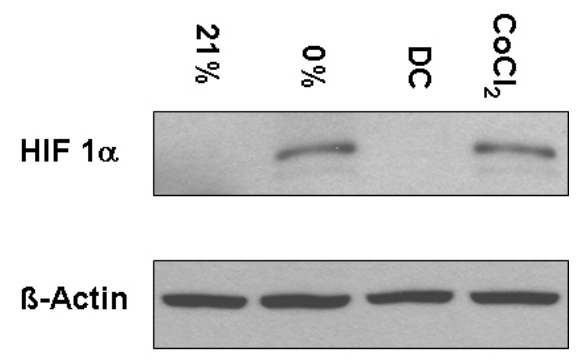

Figure 4. Western analysis was used to qualify regulation of HIF-1 $\alpha$ expression. The figure depicts upregulation of HIF-1alpha in cells exposed to hypoxia using the device, relative to the no device control (DC) and $21 \%$ oxygen. The device $0 \%$ oxygen band intensity is comparable to the $\mathrm{CoCl}_{2}$ control.

\section{DISCUSSION}

The device is effective at both controlling the temporal and spatial concentration of oxygen in a 6-well plate. Fast equilibration time and complex oxygen concentration patterning based on the microchannel design expands the experimental possibilities to include studies not possible with conventional technologies. In this study, a wide variety of different oxygen concentration profiles were achieved and many more are possible, including complex gradients and shifting oxygen regimes. As mentioned previously, the gold standard for hypoxia exposure is the hypoxic chamber, which takes hours to equilibrate, cannot achieve extremely hypoxic regimes $(<2 \%)$, and only permits a single oxygen concentration within the same cell population. However, the device presented here reduces the equilibration time orders 
of magnitude lower, yields concentrations down to $0.5 \%$ oxygen (and will be improved in later iterations of the device), and permits very complex oxygen profiles across the same well.

Despite the device's improved functionality over other hypoxia technologies, future improvements are planned including on-device custom gas mixing, media perfusion ports for slow exchange of cell media, and secondary gas ports for areas surrounding the pillar. Finally, the current device iteration was used with 6-well plates, but our lab is designing and fabricating devices for use in 12, 24, and even 96-well plates to increase experimental throughput.

\section{CONCLUSIONS}

An hypoxia device fabricated by soft lithography has been designed to adapt to the common 6-well cell culture plate. The device effectively establishes the appropriate oxygen concentration at the well bottom within minutes. Additionally, multiple oxygen concentrations and gradients can be achieved in the same well depending on the microchannel design. Both aspects are a substantial improvement over current technologies. The hypoxia insert device will greatly benefit a number of research areas requiring more control over in vitro oxygen exposure and can be utilized for experiments not feasible or possible before.

\section{ACKNOWLEDGMENT}

This project was funded by the Illinois Department of Public Health. Additionally, the authors would like to thank Parul Chansoria for help with PDMS membrane spin speed graph.

\section{REFERENCES}

1. Hagen, T., et al., Redistribution of intracellular oxygen in hypoxia by nitric oxide: effect on HIF1alpha. Science, 2003. 302(5652): p. 1975-8.

2. Huang, L.E., et al., Regulation of hypoxia-inducible factor 1alpha is mediated by an $\mathrm{O} 2$-dependent degradation domain via the ubiquitinproteasome pathway. Proc Natl Acad Sci U S A, 1998. 95(14): p. 798792.

3. Lennon, D.P., J.M. Edmison, and A.I. Caplan, Cultivation of rat marrow-derived mesenchymal stem cells in reduced oxygen tension: effects on in vitro and in vivo osteochondrogenesis. J Cell Physiol, 2001. 187(3): p. 345-55.

4. Wang, G.L., et al., Hypoxia-Inducible Factor-1 Is a Basic-Helix-LoopHelix-Pas Heterodimer Regulated by Cellular O-2 Tension. Proceedings of the National Academy of Sciences of the United States of America, 1995. 92(12): p. 5510-5514.

5. Maxwell, P.H. and P.J. Ratcliffe, Oxygen sensors and angiogenesis. Seminars in Cell \& Developmental Biology, 2002. 13(1): p. 29-37.

6. Allen, J.W., R.S. Johnson, and S.N. Bhatia, Oxygen modulation of cytochrome P450 pathways: Role of oxygen gradients and HIF-1alpha in hepatocytes in vitro. Hepatology, 2003. 38(4): p. 270a-270a.

7. Parmar, K., et al., Distribution of hematopoietic stem cells in the bone marrow according to regional hypoxia. Proceedings of the National Academy of Sciences of the United States of America, 2007. 104(13): p. 5431-5436.
8. Alvarez, M.E., et al., Reactive oxygen intermediates mediate a systemic signal network in the establishment of plant immunity. Cell, 1998. 92(6): p. 773-784.

9. Allen, J.W. and S.N. Bhatia, Formation of steady-state oxygen gradients in vitro - Application to liver zonation. Biotechnology and Bioengineering, 2003. 82(3): p. 253-262.

10. Holzer, C. and P. Maier, Maintenance of periportal and pericentral oxygen tensions in primary rat hepatocyte cultures: influence on cellular DNA and protein content monitored by flow cytometry. J Cell Physiol, 1987. 133(2): p. 297-304.

11. Uno, K., et al., Hyperoxia inhibits several critical aspects of vascular development. Developmental Dynamics, 2007. 236(4): p. 981-990.

12. Harvey, A.J., The role of oxygen in ruminant preimplantation embryo development and metabolism. Animal Reproduction Science, 2007. 98(1-2): p. 113-128.

13. Lee, H.L.T., et al., Microbioreactor arrays with integrated mixers and fluid injectors for high-throughput experimentation with $\mathrm{pH}$ and dissolved oxygen control. Lab on a Chip, 2006. 6(9): p. 1229-1235.

14. Mehta, G., et al., Quantitative measurement and control of oxygen levels in microfluidic poly(dimethylsiloxane) bioreactors during cell culture. Biomed Microdevices, 2007. 9(2): p. 123-34.

15. Tilles, A.W., et al., Effects of oxygenation and flow on the viability and function of rat hepatocytes cocultured in a microchannel flat-plate bioreactor. Biotechnology and Bioengineering, 2001. 73(5): p. 379389.

16. Vollmer, A.P., et al., Development of an integrated microfluidic platform for dynamic oxygen sensing and delivery in a flowing medium. Lab on a Chip, 2005. 5(10): p. 1059-1066.

17. Duffy, D.C., et al., Rapid Prototyping of Microfluidic Systems in Poly(dimethylsiloxane). Analytical Chemistry, 1998. 70: p. 4974.

18. Heo, Y.S., et al., Characterization and resolution of evaporationmediated osmolality shifts that constrain microfluidic cell culture in poly(dimethylsiloxane) devices. Analytical Chemistry, 2007. 79(3): p. 1126-1134.

19. Randall, G.C. and P.S. Doyle, Permeation-driven flow in poly(dimethylsiloxane) microfluidic devices. Proceedings of the National Academy of Sciences of the United States of America, 2005. 102(31): p. 10813-10818.

20. Piret, J.P., et al., $\mathrm{CoCl} 2$, a chemical inducer of hypoxia-inducible factor-1, and hypoxia reduce apoptotic cell death in hepatoma cell line HepG2. Ann N Y Acad Sci, 2002. 973: p. 443-7.

21. Allen, C.B., B.K. Schneider, and C.W. White, Limitations to oxygen diffusion and equilibration in in vitro cell exposure systems in hyperoxia and hypoxia. Am J Physiol Lung Cell Mol Physiol, 2001. 281(4): p. L1021-1027. 